\title{
HEALTH ORIENTATION AND DISORDERED EATING ATTITUDE IN WOMEN WITH POLYCYSTIC OVARY SYNDROME (PCOS)
}

\author{
Ms. Ashima Narula 1*, Dr. Mamata Mahapatra \\ 1 * Research scholar, Amity Institute of Psychology and Allied Sciences, Amity University \\ Noida, Uttar Pradesh \\ 2 Professor, Amity Institute of Psychology and Allied Sciences, Amity University \\ Noida, Uttar Pradesh \\ 1*Corresponding Author Email: narulaa74@gmail.com
}

\begin{abstract}
Reproductive medicine in the field of health psychology deals with the psychological aspects of the reproductive system and its consequences. Reproductive medicine provides an opportunity to understand the process of the menstrual cycle, pregnancy, hormonal conditions and illnesses, sexual behaviour, menopause by not merely from a biological point of view but also in light of psychological and social processes. Aline to this view, the study explored health orientation and disordered eating attitude in women diagnosed with Polycystic Ovary Syndrome (PCOS). The study captured the health-related behavior and attitude, which is a key component of the development of psychological distress in PCOS. It also examined the differences for the same in PCOS and Non-PCOS women. Total of eighty women, from the age group of 18-30 (young adults) were included in the study. The results highlighted, a significant difference among PCOS and Non-PCOS women on health image concern, health anxiety, health esteem and confidence, health expectations and health status. Finding also implied that there was a significant difference in disordered eating attitude of women diagnosed with PCOS and healthy women. These findings could have a significant implication for prevention, clinical practice and intervention in women with PCOS. The incorporation of medical and psychological actions to enhance their health-related quality of life may help these women.
\end{abstract}

Keywords- Health Orientation, Disordered Eating, Reproductive Health, Polycystic Ovary Syndrome, PCOS

\section{INTRODUCTION}

Reproductive medicine in the field of health psychology deals with the psychological aspects of the reproductive system and its consequences. It has both physical and psychological effects on women's body. In developmental lifespan, women pass through distinct developmental changes from menarche to motherhood to menopause which is associated with emotional as well as psychological challenges (Gras, R.M., 2017). Reproductive medicine provides an opportunity to understand the process of the menstrual cycle, pregnancy, hormonal conditions and illnesses, sexual behaviour, menopause by not merely from a biological point of view but also in light of psychological and social processes. 
Apart from reproductive and metabolic characteristics in condition like PCOS, women diagnosed with PCOS are at a higher and increased risk of developing psychological distress and poor wellbeing (Maron, L., Helm, M.G., et al., 2010). PCOS is one of the most common hormonal disorder among reproductive women. In India, prevalence ranges from $2.2 \%$ to $26 \%$ and seen majorly in adolescent or young women, nearly about 9.6\%. (Chaudhary, N. et al., 2011). PCOS is a complex issue causing psychological and physiological problems. Psychological factors like stress, anxiety, sexual dysfunction, depression, eating disorders, disturbed health orientation and burnout are known to be the cause as well as the symptom of PCOS (Kaplan \& Sadock, 2014).

PCOS may cause symptoms like infertility and hirsutism, which leads to generalized anxiety, body image concerns, fear of not conceiving and poor relationships. The study reports women to feel a loss of control on their own body due to excessive weight gain and hormonal imbalance (Synder, B.C., \& Patel, N., 2017). PCOS is a stigmatized issue in India, that affects women's personality, quality of life and state of wellbeing. Hirsutism, acne, obesity, infertility, pigmentation, irregular menstrual cycle impacts the mental health, which leads to clinically significant impairment in their personal, work and social functioning. In India, this dimension has not received adequate attention (Chaudhari, A.P., Mazumdar, K., \& Mehta, P.D., 2018).

A significant cause of health inequality is the psychosocial variables. These variables include cognitive distortions, several mental states, psychological features or elements of the social environment (Macleod, J., \& Smith, G.D., 2003). A wide range of factors affect health and diseases. While infectious and genetic diseases are predominant, many behavioural and psychological factors may influence overall physical wellbeing and different medical conditions (Cherry, K., 2020). Boehm (2016) summarised emerging research that points to the adjacent relations between our feelings, thoughts, actions, physical and emotional wellbeing. Seib et al. (2014) emphasized that there are deleterious impacts on our health and wellbeing from exposure to traumatic or stressful life events that we encounter at some stage of our life. And while some ill health is inevitable, and some level of stress is anticipated in our everyday lives, elevated and persistent stress may increase health-threatening actions and compromising behaviour that can impair health. It can further promote indulgence in poor lifestyle habits, physical inactivity and substance use (Rokach, A., 2019).

\section{HEALTH ORIENTATION}

Health problems, emotional disturbances and frustration are experienced by a lot of people at some point in our lives. These concerns often stem from psychological tendencies that impair the health and physical activity of an individual. However, not all psychological tendencies and factors are negatively linked to physical health; certain factors contribute to the prevention and promotion of physical health in a positive way (Snell, W.E., Johnson, G., et al., 1991). Research evidences documents that engaging in various health behaviors can be majorly dependent on individual differences, personality and environmental factors of the person (Moorman \& Matulich, 1993). 
Health orientation is a motivating factor or a force that taps into the interest of an individual in maintaining the healthy lifestyle and encourages the adoption of health behavior (DuttaBergman, M.J., 2005). The attention paid to the relevant knowledge and understanding of healthy behavior by an individual is enhanced by high degree of motivation (Celsi \& Olson, 1988; Kraft \& Goodell, 1993). Health motivated individuals are constantly pursuing accurate health information and take active interest in health issues and concerns. Health orientation manifests itself in behavioural, cognitive, emotional and attitudinal realms (Dutta-Bergman, M.J., 2005). The overall health orientation of an individual is a measure of behavioural precursors like knowledge about health, dietary behaviour and exercise behaviour.

Rimal, R.N., Flora, J.A., \& Schooler, C. (1999) conducted a study on achieving improvement in overall health orientation. It suggested that exposure to health media is associated with an increased sense of health orientation. Those who extensively use the resources and information provided are more likely to be positively related to health and encouraged to make positive lifestyle changes. Moorman \& Matulich (1993) stated that the motivation to be healthy is the representative of the degree to which the person is eager and willing to take care of his or her mental as well as physical wellbeing, unfolding health orientation as "goal-directed behaviour and arousal to engage and participative in preventive health behaviour".

Adejumo, A.O., \& Fatokun, Y. (2017) conducted a study on predictors of health expectations and health anxiety among post-salpingectomy patients in Ibadan Nigeria. The results highlighted that women with higher health expectation and anxiety were identified to have a lower level of health consciousness. Also, women with external health locus of control were highly associated with health anxiety as compared to those with internal health locus of control.

\section{DISORDERED EATING ATTITUDE AND BEHAVIOR}

Disordered eating is used to characterize a number of unhealthy eating patterns that may or may not require a particular eating disorder diagnosis (Anderson, M., 2018). It is characterized with similar low intensity and lesser frequency as those of eating disorders. Disturbed eating patterns have been shown to be linked mainly to low self-worth, body image dissatisfaction, poor self-esteem and anxiety related to various food and food groups (Pirotta, S., Barillaro, M., et al., 2019). Ching Chang, F., Mei Lee, C., et al. (2013) conducted a study on the association of thin-ideal media exposure, body dissatisfaction and disordered eating behavior among adolescents in Taiwan. The results highlighted that media pressure and body internalization significantly increased the probability of body dissatisfaction, while both restrained eating and unhealthy weight management habits contributed to media pressure and body dissatisfaction.

Disordered eating behavior and attitude are part of the spectrum of the eating disorder. Unhealthy attitude towards eating includes thinking obsessively about calories and type of food, unable to choose what to eat, craving for food to compensate psychological and emotional pain, posing unreal myths about eating beliefs and habits (Alvarenga, M.S., et al., 2010).

Psychologists describe disordered eating as unhealthy and maladaptive behavior centring around frustration with body image, restrained food, binge eating, fear of being fat or obese 
and compensatory behavior aimed at reducing the weight and minimizing the consumption of various food products (Budd, G., 2007).

\section{METHODOLOGY}

\subsection{Current Study}

The aim of the present study is to explore health orientation and disordered eating attitude in women diagnosed with Polycystic Ovary Syndrome (PCOS). The study tends to capture healthrelated behavior and attitude, which is a key component of the development of psychological distress in PCOS. Research evidence has shown the significant relationship and impact of health behavior on lifestyle, self-esteem, body-image in women PCOS as compared to healthy women. Therefore, the primary goal of this study is to identify the psychological variables associated with PCOS condition, which impacts the health-promoting behavior of an individual. Also, this study will explore the eating attitude of women and their relationship with food and ideas towards normal eating.

\section{OBJECTIVE}

- To identity the health orientation in women diagnosed with Polycystic ovary syndrome.

- To explore disordered eating behavior in women diagnosed with Polycystic ovary syndrome.

\subsection{Hypothesis}

- There will be a significant difference in health orientation of PCOS and Non-PCOS women

- There will be a significant difference in disordered eating attitude of PCOS and Non-PCOS women.

\subsection{Sample}

The total of 80 women participants were included in the study, 40 PCOS and 40 Non-PCOS women from the age group of 18-30 (young adults) were included in the study. The data was collected from healthy women and who are diagnosed with PCOS from urban domicile. Participants with any other clinically diagnosed mental disorder, reproductive health condition like ovary cancer, endometriosis and pregnancy were excluded from the study.

\section{DESCRIPTION OF THE TOOLS}

\section{- Health Orientation Scale (HOS)}

This scale was developed by Snell, W.E., Johnson, G., et al. (1991). This test measures the personality tendencies and health orientation of an individual towards physical health. It is an objective self-report measure with 50 items divided into ten dimensions:

I. Personal Health Consciousness

II. Health Image Concern

III. Health Anxiety

IV. Health Esteem and Confidence

V. Motivation to Avoid Unhealthiness

VI. Motivation for Healthiness

VII. Health Internal Control

VIII. Health External Control

IX. Health Expectations 


\section{Health Status}

The scale has high internal consistency, showing the range of 0.82 to 0.92 . The psychometric properties indicate adequate validity.

\section{- Disordered Eating Attitude Scale (DEAS)}

The scale was developed by Alvarenga, M.S., Scagliusi, F.B., \& Philippi, S.T. (2010). The purpose of the test is to identify the individual's eating attitude. The scale is appropriate for examining dysfunctional eating attitude and inconsistencies in vies, feeling, behavior and emotions in a clinical and non-clinical sample for particularly eating behavior and relationship with food. It is a 25 item self-report measure, consists of seven subscales:

I. Relationship with Food

II. Concern with Food and Weight Gain

III. Restrictive and Compensatory Behavior

IV. Food Refusal

V. Meaning of Eating

VI. Positive Feelings about Eating

VII. Idea of Normal eating

The Internal consistency reported good with the value of 0.80 , and adequate convergent and divergent validity was measured.

\section{PROCEDURE}

Women diagnosed with PCOS were approached through clinics and department of gynecology and obstetrics in hospitals of Delhi NCR. Informed consent was given before administration of the test to both the groups, i.e. PCOS and Non-PCOS participants. The participants were briefed about the purpose of the study. Demographic details including age, occupation, qualification, domicile, marital status etc. were also collected. The participants were encouraged to be honest with their answers and were informed that the data will be confidential and will be used for academic purpose only. SPSS was used for the analysis of the data collected.

\section{RESULTS}

Table 1. Showing Health Orientation Dimensions of PCOS and Non-PCOS Women

\begin{tabular}{|c|c|c|c|c|}
\hline HOS Dimensions & Groups & Mean & SD & t-value \\
\hline \multirow[b]{2}{*}{ Personal Health Consciousness } & PCOS & 12.88 & 4.15 & \multirow[b]{2}{*}{.799} \\
\hline & Non-PCOS & 12.65 & 3.70 & \\
\hline \multirow[b]{2}{*}{ Health Image Concern } & PCOS & 12.83 & 4.85 & \multirow[b]{2}{*}{$.001 *$} \\
\hline & Non-PCOS & 9.35 & 3.83 & \\
\hline \multirow[b]{2}{*}{ Health Anxiety } & PCOS & 14.38 & 4.16 & \multirow[b]{2}{*}{$.000 *$} \\
\hline & Non-PCOS & 10.10 & 4.48 & \\
\hline \multirow{3}{*}{ Health Esteem and Confidence } & PCOS & 9.48 & 3.12 & \multirow{3}{*}{$.000 *$} \\
\hline & Non-PCOS & 12.60 & 2.58 & \\
\hline & PCOS & 11.25 & 3.20 & \\
\hline
\end{tabular}




\begin{tabular}{|c|c|c|c|c|}
\hline $\begin{array}{c}\text { Motivation to Avoid } \\
\text { Unhealthiness }\end{array}$ & Non-PCOS & 10.40 & 3.57 & \multirow{2}{*}{.266} \\
\hline \multirow{3}{*}{ Motivation to Healthiness } & PCOS & 11.63 & 3.47 & \multirow{2}{*}{.411} \\
\cline { 2 - 4 } Internal Health Control & PCOS & 14.75 & 3.37 & \multirow{2}{*}{.535} \\
\cline { 2 - 4 } & Non-PCOS & 14.25 & 3.78 & \multirow{2}{*}{.744} \\
\hline \multirow{2}{*}{ External Health Control } & PCOS & 11.08 & 3.51 & \multirow{2}{*}{.} \\
\cline { 2 - 4 } Health Expectation & Non-PCOS & 11.33 & 3.30 & $000^{*}$ \\
\hline \multirow{2}{*}{ Health Status } & PCOS & 12.30 & 3.43 & \multirow{2}{*}{$.000^{*}$} \\
\hline
\end{tabular}

$*=\mathrm{p}<0.05$

The table indicates an independent sample t-test for the dimensions of health orientation among women diagnosed with PCOS and healthy women. There is a significant difference among PCOS and Non-PCOS women on health image concern, health anxiety, health esteem and confidence, health expectations and health status at 0.05 level.

Table 2. showing Disordered eating attitude among PCOS and Non-PCOS women

\begin{tabular}{|c|c|c|c|c|}
\hline DEAS Dimensions & Groups & Mean & SD & t-value \\
\hline \multirow[b]{2}{*}{ Relationship with Food } & PCOS & 33.95 & 12.18 & \multirow[b]{2}{*}{$.000 *$} \\
\hline & Non-PCOS & 27.78 & 11.35 & \\
\hline \multirow[b]{2}{*}{$\begin{array}{c}\text { Concerns about Food and Weight } \\
\text { Gain }\end{array}$} & PCOS & 11.68 & 4.34 & \multirow[b]{2}{*}{$.000 *$} \\
\hline & Non-PCOS & 6.63 & 3.09 & \\
\hline \multirow{2}{*}{$\begin{array}{c}\text { Restrictive and Compensatory } \\
\text { Practices }\end{array}$} & PCOS & 12.58 & 3.77 & \multirow[b]{2}{*}{$.000^{*}$} \\
\hline & Non-PCOS & 6.83 & 2.94 & \\
\hline \multirow[b]{2}{*}{ Feeling towards Eating } & PCOS & 10.78 & 3.37 & \multirow[b]{2}{*}{$.000 *$} \\
\hline & Non-PCOS & 5.35 & 2.34 & \\
\hline \multirow[b]{2}{*}{ Idea of Normal Eating } & PCOS & 32.45 & 8.69 & \multirow[b]{2}{*}{.370} \\
\hline & Non-PCOS & 30.90 & 6.51 & \\
\hline \multirow[b]{2}{*}{ Total DEA } & PCOS & 101.43 & 22.99 & \multirow[b]{2}{*}{$.000 *$} \\
\hline & Non-PCOS & 70.48 & 15.81 & \\
\hline
\end{tabular}

$*=\mathrm{p}<0.05$

The table indicates an independent sample t-test for the disordered eating attitude and its dimensions among women diagnosed with PCOS and healthy women. There is a significant difference between PCOS and Non-PCOS women's disordered eating attitude. Relationship with food, concern about food and weight gain, restrictive and compensatory practices and feeling towards behaviour were also significant at 0.05 level. 


\section{DISCUSSION}

The aim of the present study was to identify the health orientation and disordered eating attitude of women diagnosed with Polycystic Ovary Syndrome. Aline to this view, empirical evidences suggests that an individual's psychological tendencies are positively associated with physical health (Snell, W.E., Johnson, G., et al., 1991). Women diagnosed with PCOS are especially vulnerable to increased perceived stress, body dissatisfaction, disordered eating, health anxiety and depression. As potential contributors to adverse psychological outcome in PCOS women, factors such as infertility, elevated body mass index, metabolic disorders have been proposed (Pirotta, S., Barillaro, M., et al., 2019). Dakanalis, A., Clerici, M., et al. (2017) highlighted the higher risk of disordered eating and eating disorder was associated with younger women generation, in PCOS. Frequent comparison of one's body with ideal internalization encourage the idea of social expectations and leads to guilt, shame, anxiety and engagement in unhealthy behaviors.

The quantitative analysis highlighted the difference between health orientation and disordered eating attitude among women diagnosed with PCOS and healthy women. As mentioned in table 1 , there was a significant difference in certain dimensions of health orientation. PCOS women scored higher on subscale "health image concern" as they have a chronic inclination of the external, visible impressions to be conscious of what their physical fitness means to others. Women who are highly concerned with health image are extremely concerned about the impression of others created by their physical health. In comparison, PCOS was associated with anxiety and nervousness concerning one's physical health on the dimension, "health anxiety". Compared to healthier women, depression and anxiety are more prevalent in patients with PCOS. Obesity and insulin resistance can be linked with health anxiety (Cinar, N., Kizilarslanoglu, M.C., et al., 2011).

Another dimension of health orientation, "Health esteem and confidence" was also higher in PCOS women. Healthier women were more confident and tend to evaluate and perceive their health positively. Bazarganipour, F., Ziaei, S., et al. (2014) conducted a study on health-related quality of life in patients with Polycystic Ovary Syndrome. The results highlighted that selfesteem, confidence, body appearance, and sexual dysfunction had the greatest influence of PCOS symptoms on HR-QOL disability in patients. The two motivation-based subscales of health orientation, "motivation to avoid unhealthiness" and "motivation to healthiness" were not of significant difference in both the groups. These subscales embrace the need to escape unhealthy behavior to avoid being in exceptionally poor health and concerned with motivating tendency to maintain oneself in good physical condition. Health-promoting behavior enables an individual to initiate and control over their physical health to ultimately improve healthrelated quality of life with or without being diagnosed with any medical condition (Mirghafourvand, M., Charandabi, S.M., et al., 2017).

External health control and Internal health control were also insignificant. On the ninth dimension, "health expectations", PCOS women were more concerned about other's impression and expectations about their health status and condition. Symptoms like facial hair and 
pigmentation as perceived as socially undesirable and are one of the most distressing symptoms of PCOS. This leads to women challenging their opinions of themselves as feminine and struggling to deal with expectations of others (Mccook, J., bailey, B.A., et al., 2014). The health orientation scale's final subscale was concerned with the contemporary health status of an individual. Healthy women scored higher on this, as individual higher on this subscale rate themselves as well-exercised and in decent physical shape.

Hypothesis 2 was accepted as there is a significant difference in the disordered eating attitude of women diagnosed with PCOS and healthy women. Lee, I., Cooney, L.G., et al. (2019) conducted a study on increased odds of disordered eating in polycystic ovary syndrome. The results suggested that women with PCOS are at increased odds of developing disordered eating and unhealthy eating behavior. Body image and physical symptoms in PCOS are crucial for predicting eating behavior and sociocultural internalization of the standard of thinness and body dissatisfaction (Karacan, E., Caglar, G.S., et al., 2014). The dimensions of disordered eating attitude scale-relationship with food, concern about food and weight gain, restrictive and compensatory practices and feeling towards eating exhibited significant difference among PCOS and Non-PCOS women. The final dimension "idea of normal eating" was insignificant. Hajivandi, L., Noroozi, M., et al. (2020) explored the food habits in overweight and obese adolescents' girls with polycystic ovary syndrome (PCOS). The qualitative analysis extracted that PCOS women were indulged in a high intake of unhealthy food, high intake of fatty and salty snacks and sugar-rich food products. Lower consumption of fibre-rich food and nutritious food was also observed. The most frequently picked characteristics in PCOS are trouble losing weight, erratic menstrual periods, infertility and excess hair development (Helm, M. G., Teede, H., et al., 2016). Mizgier, M., Jarzabek-Bielecka, G., et al. (2020) looked upon the risk factors of overweight and obesity-related to diet and disordered eating attitudes in adolescent girls with clinical features of polycystic ovary syndrome. The findings highlighted that being overweight or obese is significantly associated with disordered eating attitude and the type and quantity of protein intake.

Mental health problems comorbid with physical illness or disease is highly associated with health-related quality of life (Farhangi, M.A., Dehghan, P., et al., 2018). Unhealthy eating pattern or disordered eating are important determinants of mental and physical health problems like depression anxiety, burnout, hypertension, thyroid, disturbed menstrual cycle and hormonal imbalance.

\section{CONCLUSION AND FUTURE IMPLICATIONS}

The study explored the health orientation and disordered eating attitude among women diagnosed with polycystic ovary syndrome. PCOS is highly prevalent and chronic condition in women with reproductive age, so assessing health orientation and eating attitude as a way to measure their psychological tendencies alongside medical treatment may be significant. These findings could have major implications for prevention, clinical practice and intervention in women with PCOS. The incorporation of medical and psychological actions to enhance their health-related quality of life may help these women. 


\section{REFERENCES}

1. Sanchez-Ferrer, M.L., Adoamnei, E., et al. (2020). Health-related quality of life in women with polycystic ovary syndrome attending to a tertiary hospital in Southeastern Spain: a casecontrol study. Health and Quality of life outcomes, 18, 232. https://doi.org/10.1186/s12955020-01484-z

2. Hajivandi, L., Noroozi, M., et al. (2020). Food habits in overweight and obese adolescent girls with Polycystic ovary syndrome (PCOS): A qualitative study in Iran. BMC Pediatrics, 20, 277. https://doi.org/10.1186/s12887-020-02173-y

3. Karacan, E., Caglar, G.S., et al. (2014). Body satisfaction and eating attitudes among girls and young women with and without Polycystic ovary syndrome. Journal of pediatric and adolescent gynecology, 27(2), 72.77. https://doi.org/10.1016/j.jpag.2013.08.003

4. Lee, I., Cooney, L.G., Saini, S., et al. (2019). Increased odds of disordered eating in polycystic ovary syndrome: a systematic review and meta-analysis. Eating and weight disorders- studies on anorexia, bulimia and obesity. 24, 787-797. https://doi.org/10.1007/s40519-018-0533-y

5. McCook, J., Bailey, B., et al. (2015). Differential contributions of polycystic ovary syndrome (PCOS) manifestations to psychological symptoms. Journal of behavioral health services \& research, 42(3), 383-394. https://doi.org/10.1007/s11414-013-9382-7

6. Cinar, N., Kizilarslanoglu, M.C., et al. (2011). Depression, anxiety and cardiometabolic risk in polycystic ovary syndrome. Human reproduction, 26(12), 3339-3345. https://doi.org/10.1093/humrep/der338

7. Ching-Chang, F., Mei-Lee, C., et al. (2013). Association of thin ideal media exposure, body dissatisfaction and disordered eating behavior among adolescents in Taiwan. Eating Behaviors, $14(3)$, 382-385. https://www.researchgate.net/deref/http\%3A\%2F\%2Fdx.doi.org\%2F10.1016\%2Fj.eatbeh.20 13.05.002

8. Rokach, A. (2019). Health, illness and psychological factors affecting them. The journal of Psychology, 153(1), 1-5. https://doi.org/10.1080/00223980.2018.1548202

9. Snell, W.E., Johnson, G., Lloyd, P.J., \& Hoover, M.W. (1991). The health orientation scale: A measure of psychological tendencies associated with health. European journal of personality, 5, 169-183. https://psycnet.apa.org/doi/10.1002/per.2410050208

10. Rimal, R.N., Flora, J.A., \& Schooler, C. (1999). Achieving improvements in overall health orientation: Effects of campaign exposure, information seeking, and health medical use. Communication Research, 26(3), 322-348. https://doi.org/10.1177\%2F009365099026003003

11. Farhangi, M.A., Dehghan, P., \& Jahangiry, L. (2018). Mental health problems in relation to eating behavior pattern, nutrient intake and health related quality of life among Iranian female adolescents. PLoS ONE, 13(4), 1-15. https://doi.org/10.1371/journal.pone.0195669

12. Adejumo, A.O., \& Fatokun, Y. (2017). Predictors of health expectation and health anxiety among post-salpingectomy patients in Ibadan Nigeria. Journal of reproductive and infant psychology, 35(2), 196-206. https://doi.org/10.1080/02646838.2016.1256472

13. Macleod, J., \& Smith, G.D. (2003). Psychosocial factors and public health: a suitable case for treatment? Journal of Epidemiology and Community Health, 57, 565-570. https://doi.org/10.1136/jech.57.8.565 
14. Dutta-Bergman, M.J. (2005). The relation between health-orientation, provider-patient communication, and satisfaction: An individual-difference approach. Health Communication, 18(3), 291-303. https://doi.org/10.1207/s15327027hc1803_6

15. Alvarenga, M.S., et al. (2010). Development and validity of the disordered eating attitude scale (DEAS). Perceptual and Motor skills, 110(2), 379-395. https://doi.org/10.2466\%2Fpms.110.2.379-395

16. Pirotta, S., Barillaro, M., et al. (2019). Disordered eating behaviors and eating disorders in women in Australia with and without polycystic ovary syndrome: A cross sectional study. $\begin{array}{llll}\text { Journal of } & \text { clinical } & \text { medicine, } & \end{array}$ https://www.researchgate.net/deref/http\%3A\%2F\%2Fdx.doi.org\% 2 F10.3390\%2Fjcm810168 2

17. Budd, G. (2007). Disordered eating: Young women's search for control and connection. Journal of child and adolescent psychiatric nursing, 20(2), 96-106. https://doi.org/10.1111/j.1073-6077.2007.00091.x

18. Mizgier, M., Jarzabek-Bielecka, G., et al. (2020). Risk factors of obesity and weight related to diet and disordered eating attitudes in adolescent girls with clinical features of polycystic ovary syndrome. Journal of Clinical medicine, 9(9), 3041. https://doi.org/10.3390/jcm9093041

19. Maron, L., Helm, M.G., et al. (2010). Polycystic ovary syndrome: A biopsychosocial understanding in young women to improve knowledge and treatment options. Journal of $\begin{array}{lllll}\text { Psychosomatic Obstetrics } \quad \text { \& } \quad \text { Gynecology, } & 31(1), \quad 31 .\end{array}$ https://doi.org/10.3109/01674820903477593

20. Choudhary, N., Padmalatha, V., et al. (2011). Prevalence of polycystic ovarian syndrome in adolescents. Journal of paediatric and adolescent gynecology, 24(4), 223-227. https://doi.org/10.1016/j.jpag.2011.03.002

21. Sadock, B. J., Sadock, V. A., \& Ruiz, P. (2015). Kaplan \& Sadock's synopsis of psychiatry: Behavioral sciences/clinical psychiatry (Eleventh edition.). Philadelphia: Wolters Kluwer.

22. Chaudhari, A.P., Mazumdar, K., \& Mehta, P.D. (2018). Anxiety, depression, and quality of life in women with polycystic ovary syndrome. Indian journal of psychological medicine, 40(3), 239.

https://www.researchgate.net/deref/http\%3A\%2F\%2Fdx.doi.org\%2F10.4103\%2FIJPSYM.IJ PSYM_561_17

23. Cherry, K. (2020, June 28). Studying health psychology and illness. https://www.verywellmind.com/what-is-health-psychology-2794907

24. Seib, S., Whiteside, E., Lee, K., Humphreys, J., Dao Tran, T. H., Chopin, L., \& Anderson, D. (2014). Stress, lifestyle, and quality of life in midlife and older Australian women: Results from the stress and the health of women study. Women's Health Issues, 24(1), 43-52. doi:10.1016/ j.whi.2013.11.004.

25. Moorman, C. \& Matulich, E. (1993). A model of consumer's preventive health behaviors: The role of health motivation and health ability. Journal of consumer research, 20(2), 208-228. https://doi.org/10.1086/209344

26. Celsi, R. L., \& Olson, J. C. (1988). The role of involvement in attention, and comprehension processes. Journal of Consumer Research, 15, 210-224. https://doi.org/10.1086/209158

27. Dakanalis, A., Clerici, M., et al. (2017). Risk and maintenance factors for young women's DSM 5 eating disorders. Europe PMC, 20(6), 721-731. https://doi.org/10.1007/s00737-017-0761-6 
28. Dakanalis, A., Clerici, M. \& Stice, E. (2019). Prevention of eating disorders: current evidencebase for dissonance-based programmes and future directions. Eating \& Weight DisordersStudies on Anorexia, Bulimia and obesity, 24, 597-603. https://doi.org/10.1007/s40519-01900719-3

29. Bazarganipour, F., Ziaei, S., et al. (2014). Health-related quality of life in patients with polycystic ovary syndrome (PCOS): A model-based study of predictive factors. The journal of sexual medicine, 11(4), 1023-1032. https://doi.org/10.1111/jsm.12405

30. Mirghafourvand, M., Charandabi, S.M., et al. (2017). Realtionship between health promoting lifestyle and quality of life in women with polycystic ovary syndrome. International journal of women's health and reproduction sciences, 5(4), 318-323. DOI: 10.15296/ijwhr.2017.54

31. Karacan, E., Caglar, G.S., et al. (2014). Body satisfaction and eating attitudes among girls and young women with and without polycystic ovary syndrome. Journal of Pediatric and adolescent gynecology, 27(2), 72-77. https://doi.org/10.1016/j.jpag.2013.08.003 